\title{
Fine structure and spin quantum beats in InP quantum dots in a magnetic field
}

\author{
I. A. Yugova* and I. Ya. Gerlovin ${ }^{\dagger}$ \\ Institute of Physics, St.-Petersburg State University, St.-Petersburg, 198504, Russia
}

\author{
V. G. Davydov, I. V. Ignatiev, ${ }^{\ddagger}$ I. E. Kozin, ${ }^{\S}$ H. W. Ren, ${ }^{\mathbb{I}}$ M. Sugisaki, ${ }^{* *}$ S. Sugou, ${ }^{\dagger \dagger}$ and Y. Masumoto ${ }^{\ddagger *}$ \\ Single Quantum Dot Project, ERATO, JST, Japan
}

(Received 13 March 2002; revised manuscript received 27 June 2002; published 16 December 2002)

\begin{abstract}
The paper reports on quantum beats observed in the photoluminescence kinetics of a single layer of the InP self-assembled quantum dots in a magnetic field. It is found that the beats arise only after removal of excess charges from the quantum dots by an external electrical bias. The quantum beats are shown to be related to the interference of the excitonic fine-structure states split by the magnetic-field. The dependences of the beat characteristics on the magnetic-field strength and orientation are studied. Theoretical analysis based on a model spin Hamiltonian has allowed us to describe adequately the shape of the oscillating component of the signal. We have determined the values of the electron $g$-factor components and estimated the spread and the mean value of the hole $g$ factor, as well as of the electron-hole exchange splitting parameters.
\end{abstract}

DOI: 10.1103/PhysRevB.66.235312

PACS number(s): 73.21.-b, 71.70.Ej, 78.55.Cr, 78.67.Hc

\section{INTRODUCTION}

Spin dynamics of carriers and excitons in the lowdimensional semiconductor heterostructures attracts nowadays a particular interest in view of potential feasibility of the logic and computer memory elements based on the effects of optical spin orientation. ${ }^{1-3}$ The most promising, in this respect, are the structures with quantum dots (QD's) whose spin states are characterized by a high stability. ${ }^{4}$ The main drawback of such structures is a large inhomogeneous broadening of their energy states, resulting from a strong spread of the QD parameters. The inhomogeneous broadening hampers getting information about spin-related structure of the excitonic states (fine structure) and makes impossible analysis of the spin dynamics. The inhomogeneous broadening can be eliminated using the single QD spectroscopy technique. ${ }^{5-7}$ This technique, however, provides information about individual QD's rather than about the ensemble as a whole.

An efficient way to determine the ensemble-averaged fine-structure parameters is considered to be detection of the quantum beats (QB's) associated with interference between the spin states. This method is attractive due to its ability to detect small splittings (fractions of meV) hidden within inhomogeneously broadened excitonic transitions. The quantum beats technique is widely used for studying the fine structure and Zeeman splitting in quantum wells and superlattices. ${ }^{8-13}$

One could expect that the QB technique may be also successfully applied to studies of QD's. Indeed, there are several publications where the QB's in QD's were observed. But these observations were made only under specific experimental conditions. In particular, in the absence of the magnetic field, the beats between the exciton fine-structure states, split by the anisotropic exchange interaction, were observed in Ref. 16. Another example is the QB's between the finestructure sublevels of the negatively charged exciton (trion). ${ }^{17}$ Besides, the beats related to the free-electron spin precession in a transverse magnetic field were detected in
Refs. 14 and 15. At the same time, no information about the QB's between the Zeeman sublevels of the excitonic states in QD's is available in the literature.

Until recently, the reasons why observation of the spin beats in QD's are hampered were obscure. As a rule, the absence of the QB's is associated with a great spread of the Zeeman splittings in the inhomogeneous ensemble of QD's. In our opinion, however, there is one more, perhaps not less important, reason related to the fact that QD's in most structures are charged. This circumstance was pointed out by many authors. ${ }^{5,6,17-20}$ Even the presence of a single excess charge drastically complicates the excited-state fine structure. ${ }^{17}$ As a result, the polarized light excites several, rather than only two, sublevels split by the magnetic field. In this case, the beats at different frequencies interfere and the oscillations of the resulting signal virtually vanish, which was observed experimentally. ${ }^{17}$ So, observation of the QB's on the fine-structure Zeeman components is possible only in the ensemble of neutral QD's.

In this paper, we report on a study of kinetics of resonance photoluminescence (PL) of the InP QD's in a magnetic field. We have found that discharging the initially charged QD's by an external electric field, indeed, gives rise to strong QB's. Polarization characteristics of these beats differ from standard characteristics of the beats in the excitonic Zeeman doublet. The frequency of the beats was found to be dependent on the magnetic-field orientation. A theoretical analysis of the experimental data performed with the use of a model spin Hamiltonian has allowed us to identify the fine structure of the excitonic states in the system under study, to determine the longitudinal and transverse components of the electronic $g$ factor, and to estimate the spread of the hole $g$ factor and electron-hole exchange interaction parameters.

\section{EXPERIMENTAL DETAILS}

We studied a heterostructure with a single layer of the InP QD's sandwiched between the $\operatorname{In}_{0.5} \mathrm{Ga}_{0.5} \mathrm{P}$ barrier layers. The sample was grown by the gas source molecular-beam epitaxy 

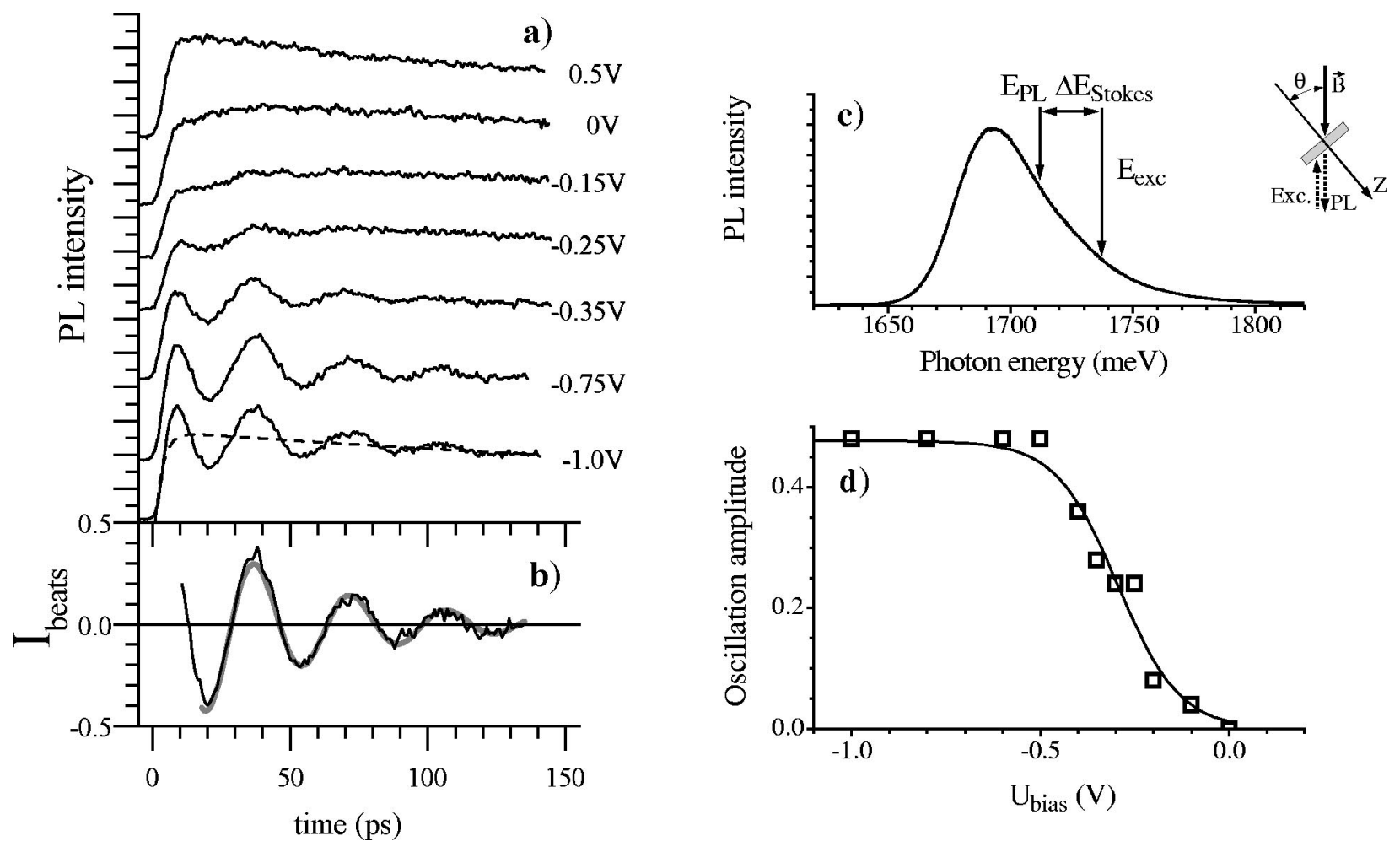

FIG. 1. (a) PL kinetics in the circular co-polarization for different biases applied to the top surface of the sample, indicated against each curve. $B=2 \mathrm{~T}, \theta=40^{\circ}, \Delta E_{\text {Stokes }}=44.5 \mathrm{meV}$. The dashed line shows fitting of the smoothly varying background by function (2) with the parameters $\tau_{r}=2.8 \mathrm{ps}, \tau_{P L}=456.6 \mathrm{ps}$. (b) Oscillating part of the PL signal normalized to $I_{s}$ (noisy curve) and its fitting by formula (1) (thick gray line). (c) PL spectrum of the QD's. The arrows show the photon energies for the exciting and detected light spaced by the Stokes shift $\Delta E_{\text {Stokes }}$. Geometry of the experiment is shown schematically in the inset. (d) Dependence of the beat amplitude $I_{\text {beats }}(0)$ on the bias (open squares) and the fitting by the function $y=y_{0}\left\{1-\operatorname{erf}\left[\left(U-U_{1 / 2}\right) /(0.6 \Delta U)\right]\right\}$ (solid line), where $U_{1 / 2}=U_{0}-\Delta U / 2, U_{0}=-0.2 \mathrm{~V}, \Delta U$ $=0.22 \mathrm{~V}$, and $\operatorname{erf}(x)$ is the error function. The values of the parameters $U_{0}$ and $\Delta U$ are taken from Ref. 17.

on an $n^{+}$GaAs substrate. Details of the growth procedure and sample characterization are given in Refs. 21-24. The areal density of the QD's is about $10^{10} \mathrm{~cm}^{-2}$. The average base diameter of the QD's is $\approx 40 \mathrm{~nm}$ and the height is $\approx 5 \mathrm{~nm}$. To control the charge of the QD's, the sample was provided with a semitransparent indium tin oxide Shottky contact on the top surface and an Ohmic contact on the other one. The thickness of the undoped epitaxial layers, to which the electric voltage was applied, was about $0.5 \mu \mathrm{m}$.

In kinetic studies, the luminescence was excited by 3-ps pulses of a Ti:sapphire laser within the PL band (quasiresonant excitation) as shown in Fig. 1(c). The PL kinetics was measured with a time resolution of 6 ps using a $0.25-\mathrm{m}$ subtractive double monochromator and a streak camera. The measurements were made in a cryostat with a superconducting magnet in the fields up to $7 \mathrm{~T}$. The design of the cryostat allowed us to excite the sample and to detect its emission either along the magnetic-field direction (the Faraday configuration) or across the field (the Voight configuration). The PL was detected in the backscattering geometry as shown in the inset of Fig. 1. To study the beats at different angles $\theta$ between the growth axis $z$ of the structure and direction of the magnetic field $B$, the sample was rotated around the vertical axis.

\section{EXPERIMENTAL RESULTS}

As has been shown experimentally, the PL pulse shape in a magnetic field is strongly affected by the applied bias. At zero and positive bias, the shape is smooth while at negative bias, the PL kinetics exhibits pronounced intensity oscillations as shown in Fig. 1(a). The oscillating part of the signal $I_{\text {beats }}$ can be determined by subtracting the smoothly varying background $I_{s}$ from the total signal $I_{P L}$ and normalizing to $I_{s}: I_{\text {beats }}=\left(I_{P L}-I_{s}\right) / I_{s}$. The oscillations can be well approximated by a simple equation,

$$
I_{\text {beats }}(t)=I_{\text {beats }}(0) \exp (-t / \tau) \cos (\omega t)
$$

as shown in Fig. 1(b). Here, $I_{\text {beats }}(0), \omega$, and $\tau$ are the amplitude, frequency, and decay time of the oscillations, respectively.

The background can be approximated by the function

$$
I_{s}=I_{0}\left[\exp \left(-t / \tau_{P L}\right)-\exp \left(-t / \tau_{r}\right)\right]
$$

The parameters $\tau_{r}$ and $\tau_{P L}$ characterize the PL rise and decay times, respectively. The PL rise is related to relaxation of the hot photogenerated carriers to the radiative energy level. This process was studied in detail in Ref. 23. The PL decay results from radiative recombination of the electron-hole 
pairs and nonradiative loss of the excitations. At negative bias, the nonradiative loss is due to the hole tunneling process as shown in Ref. 23.

The analysis of the experimental data has shown that the oscillation frequency as well as the oscillation decay time do not depend on the applied voltage. At the same time, the oscillation amplitude $I_{\text {beats }}(0)$ rapidly increases with increasing negative bias beginning from $U_{0}=-0.15 \mathrm{~V}$, while at $U_{0}<-0.5 \mathrm{~V}$ the growth of the amplitude is saturated [see Fig. 1(d)]. We attribute this behavior of the oscillations to variations in the QD's charge state.

The InP QD's grown on the $n$-doped GaAs substrate are known to be usually charged. ${ }^{20}$ The presence of the charges is related to the fact that the lowest electronic level of the QD's is positioned below the Fermi level of the doped substrate. Application of a negative voltage to the top surface of the sample removes the excess carriers from the dots and thus renders them neutral. ${ }^{20,22}$ The magnitude of the voltage at which most dots become neutral depends on the mean size of the dots and equals, in the structure under study, $U_{\text {bias }}$ $\leqslant-0.5 \mathrm{~V} .{ }^{17}$ As seen from Fig. 1(d), the oscillation amplitude becomes the greatest exactly at this voltage. Such a correlation between the beat amplitude and the charge state of the QD's allowed us to conclude that observation of the QB's in a magnetic field is possible only for QD's with no excess charge. The presence of a finite interval $\Delta U$, in which the value of $I_{\text {beats }}(0)$ increases, is likely to be caused by a spread of heights of the potential barriers in the QD's ensemble. $^{25}$

We studied behavior of the oscillations in different experimental conditions. The results of the study have shown that the oscillations are observed under excitation within or above the PL band of the QD's and under PL detection with the Stokes shifts, $\Delta E_{\text {Stokes }}$, up to $\Delta E_{\text {Stokes }}=70 \mathrm{meV}$. The oscillation frequency does not depend on the exciting photon energy and slightly decreases with decreasing photon energy of the detected PL. At the same time, the oscillation amplitude essentially depends on the Stokes shift between the PL and exciting light frequencies. The most intense oscillations are observed at the Stokes shift $\Delta E_{\text {Stokes }}=45 \mathrm{meV}$, which corresponds to the energy of the longitudinal optical (LO) phonons in the InP QD's. ${ }^{23}$ All the data presented below are obtained for this value of the Stokes shift. The spectral dependence of the oscillation amplitude is discussed in detail elsewhere. ${ }^{26}$

Polarization characteristics of the oscillations appeared to be rather curious. It was found (see Fig. 2) that the most intense oscillations were observed under circularly polarized excitation with detection of the PL in the same polarization (co-polarized PL). Almost no oscillations were observed in the cross-polarized PL. Under the linearly polarized excitation, the oscillations in kinetics of the linearly co- and crosspolarized PL have the same phase. Their frequency coincides with that in circular polarization, but their amplitude is substantially smaller. It is noteworthy that the antiphase oscillations in the linear co- and cross- polarized PL, typical for the QB's of split Zeeman sublevels, ${ }^{8,27}$ were not observed in our experiments.

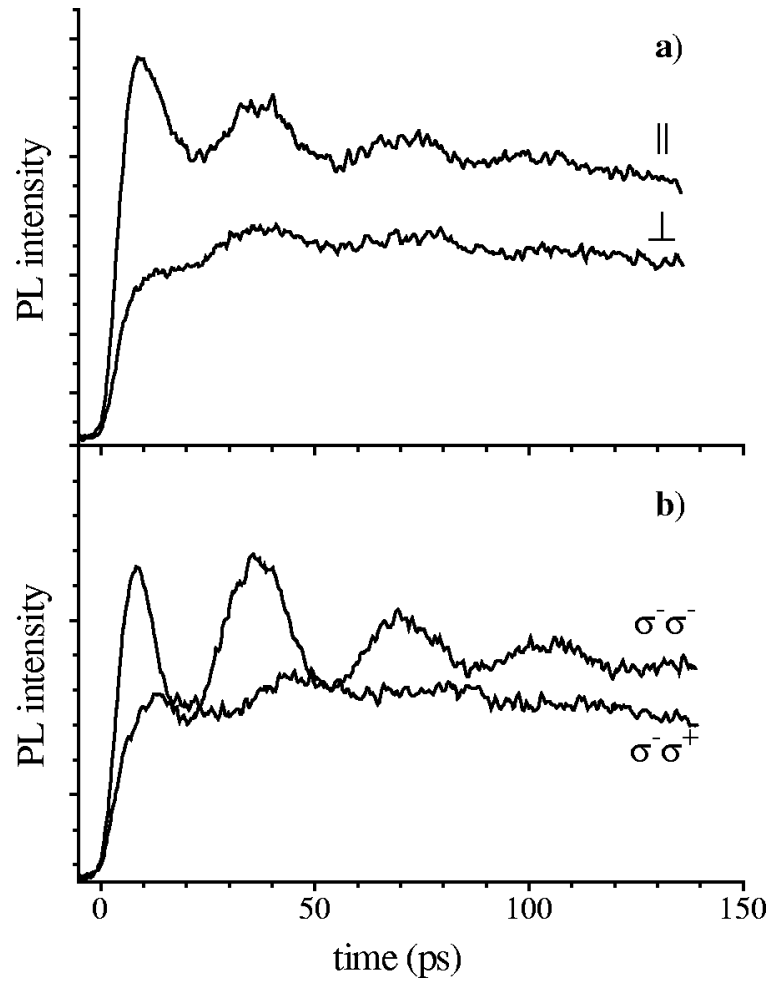

FIG. 2. PL kinetics in different polarizations (indicated against each curve). Symbols $\|$ and $\perp$ denote the co- and cross-linear polarizations, respectively; $\sigma^{+}$and $\sigma^{-}$denote circular polarization of the right and left helicities, respectively. In the case of circular polarization the first symbol denotes polarization of the excitation and the second the PL polarization. Experimental conditions are $B$ $=2 \mathrm{~T}, \theta=40^{\circ}, \Delta E=45 \mathrm{meV}$, and $U_{\text {bias }}=-0.75 \mathrm{~V}$.

To identify the nature of the oscillations, we studied the effect of the magnetic-field strength and orientation on the shape of the oscillations. Figure 3 shows dependence of the oscillations on the magnetic-field strength. One can easily see that an increase in the field is accompanied by an increase in the frequency of the oscillations. Fitting the oscillating part of the signal by Eq. (1) has allowed us to find that, in the range of 1-4 $\mathrm{T}$, the oscillation frequency is directly proportional to the field strength as shown in Fig. 3(b). In the fields below $1 \mathrm{~T}$, the period of the beats becomes longer than the decay time, and the oscillation frequency cannot be determined.

The behavior of the oscillations versus the magnetic-field orientation is found to be highly unusual. In the Faraday configuration $\left(\theta=0^{\circ}\right)$, typical for observation of the Zeeman splitting in low-dimensional structures, ${ }^{8,27,28}$ the oscillations are totally absent both in the linear and circular polarizations. Deviation of the magnetic field from the $z$ axis is accompanied by appearance of the oscillations [see Fig. 4(a)]. Within the range of the angles $20^{\circ}<\theta<60^{\circ}$, the oscillation amplitude virtually does not depend on the angle, while at higher $\theta$, the amplitude substantially decreases. The oscillation frequency essentially increases with increasing angle $\theta$, i.e., with increasing transverse component of the magnetic field, as shown in Fig. 4(b).

Since the oscillations described above are observed only 

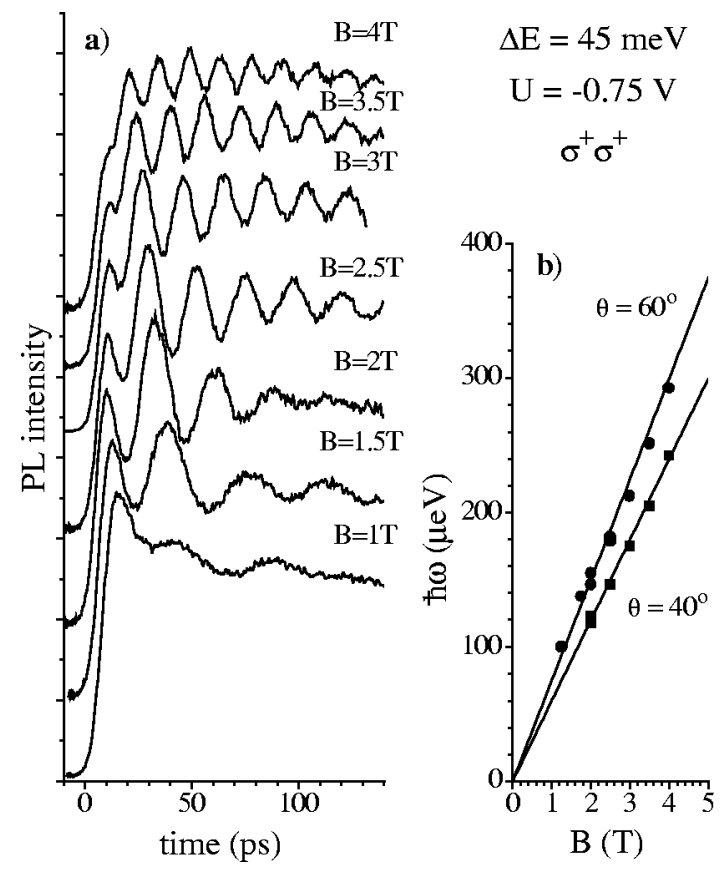

FIG. 3. (a) PL kinetics for different values of the magnetic field (indicated against each curve) for $\theta=60^{\circ}$. (b) Dependence of the oscillation frequency (in energy units) on the magnetic-field strength for two different angles $\theta$. The symbols are the experimental data and solid lines are the theoretical fits.
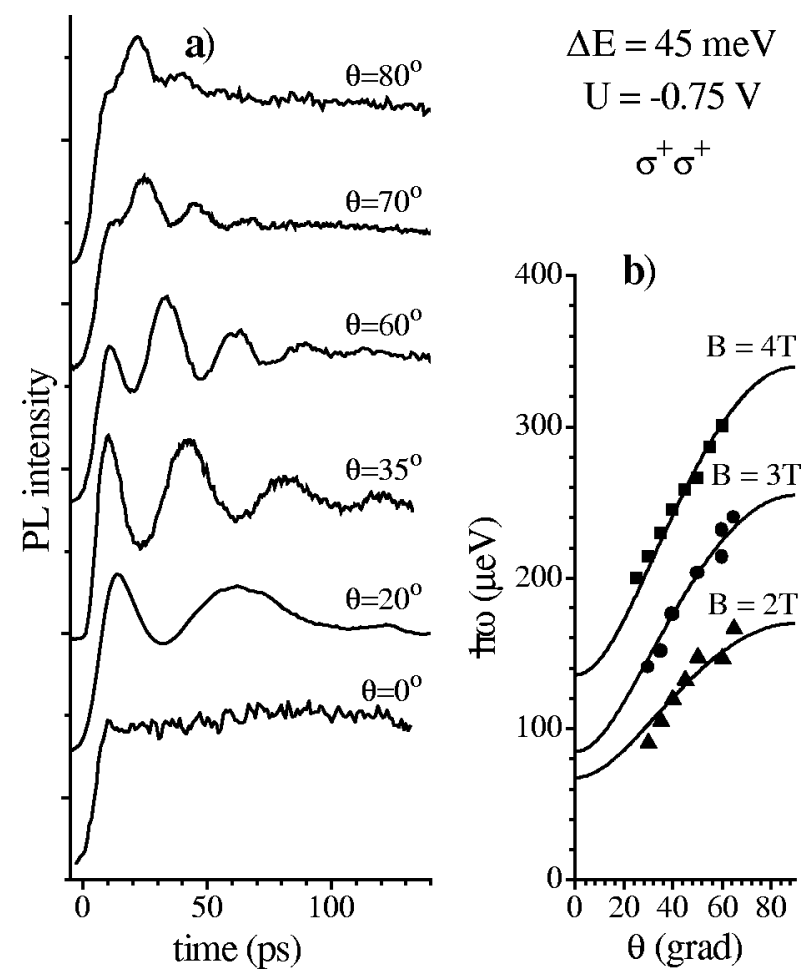

FIG. 4. (a) PL kinetics at different angles $\theta$ (indicated against each curve), $B=2 \mathrm{~T}$. (b) Dependence of the oscillation frequency (in energy units) on the angle $\theta$ for three values of the magnetic field. Symbols are the experimental data and solid lines are the theoretical fits. in the presence of a magnetic field, it is naturally to ascribe them to quantum beats between components of the excitonic fine structure split by the magnetic field. For analysis of the experimental data, we use the theoretical model of the excitonic fine structure, presented in the next section.

\section{FINE-STRUCTURE MODEL}

\section{A. Spin Hamiltonian}

We analyzed characteristics of the fine-structure components using a theoretical model of the spin Hamiltonian of the electron-hole pair in a magnetic field similar to that presented in Refs. 28-30. Within the framework of this model, the Hamiltonian of spin states of the electron-hole pair in a bulk material can be presented in the form

$$
H_{e x}=H_{e}+H_{h}+H_{e-h} .
$$

The first term, $H_{e}$, describes the Zeeman splitting of the electronic states:

$$
H_{e}=\mu_{B} g_{e} \sum_{i=x, y, z} S_{e, i} B_{i},
$$

where $\mu_{B}$ is the Bohr magneton, $g_{e}$ is the electron $g$ factor, and $S_{e, i}$ and $B_{i}$ are the Cartesian components of the electron spin and magnetic field, respectively. The second term, $H_{h}$, in Eq. (3) describes Zeeman splitting of the hole states:

$$
H_{h}=-\mu_{B} \sum_{i=x, y, z}\left(k J_{h, i}+q J_{h, i}^{3}\right) B_{i},
$$

where $k$ and $q$ are the Zeeman splitting constants and $J_{h, i}$ is the $i$ component of total angular momentum of the hole. The last term in Eq. (3), $H_{e-h}$, describes exchange interaction between the electron and hole spins:

$$
H_{e-h}=-\sum_{i=x, y, z}\left(a J_{h, i} S_{e, i}+b J_{h, i}^{3} S_{e, i}\right) .
$$

Here, $a$ and $b$ are the spin-spin coupling constants.

In the InP-type semiconductors, the ground excitonic state is formed by an electron with the spin $S_{e}=\frac{1}{2}$ and a hole with the total angular momentum $J_{h}=\frac{3}{2}$. The size quantization along the growth axis $z$ realized in the quasi-twodimensional systems (quantum wells and superlattices) is accompanied by splitting of the hole state into the light hole (LH) $\left(J_{h, z}= \pm \frac{1}{2}\right)$ and heavy hole $(\mathrm{HH})$ states $\left(J_{h, z}= \pm \frac{3}{2}\right)$. The value of the splitting substantially exceeds typical Zeeman splittings in magnetic fields used. ${ }^{31}$ This allows one to independently analyze the fine structure of the $\mathrm{LH}$ and $\mathrm{HH}$ excitons. As a rule, in the low-dimensional structures, the lowest energy state is that of the heavy hole. For this reason, the analysis presented below is restricted to the fine structure of the electron-HH pair or $\mathrm{HH}$ exciton.

The spin Hamiltonian of the $\mathrm{HH}$ exciton can be reduced to the fairly simple form ${ }^{29}$

$$
H_{e x}=\sum_{i=x, y, z}\left[\mu_{B}\left(g_{e, i} S_{e, i}-g_{h, i} \widetilde{S}_{h, i}\right) B_{i}-c_{i} S_{e, i} \widetilde{S}_{h, i}\right]
$$


where $\widetilde{S}_{h}$ is the effective spin of the hole with the components $\pm \frac{1}{2}$ corresponding to the components $J_{h, z}=\mp \frac{3}{2}, g_{e, i}$, and $g_{h, i}$ are the components of the electron and hole $g$ factors, respectively, and $c_{i}$ is the spin-spin coupling constant. The relation of the quantities $g_{h, i}$ and $c_{i}$ with the coefficients $k, q, a$, and $b$ [see Eqs. (5) and (6)] is given by the formulas:

$$
\begin{gathered}
g_{h, z}=-3 k_{z}-27 q_{z} / 4 ; \quad g_{h, x}=3 q_{x} / 2 ; \quad g_{h, y}=3 q_{y} / 2 ; \\
c_{z}=-3 a_{z}-27 b_{z} / 4 ; \quad c_{x}=3 b_{x} / 2 ; \quad c_{y}=3 b_{y} / 2 .
\end{gathered}
$$

In the framework of the model under consideration, the eigenstates of the excitonic fine structure are characterized by projections of the total angular momentum of the exciton $\mathbf{J}=\mathbf{S}_{\mathbf{e}}+\mathbf{J}_{\mathbf{h}}$ upon the quantization axis. Accordingly, the states of the $\mathrm{HH}$ exciton $\left\{\varphi_{i}\right\}$ can be denoted as $|+1\rangle,|-1\rangle$, $|+2\rangle$, and $|-2\rangle$. In conformity with the selection rules, the optical transitions into the states with $J_{z}= \pm 1$ are allowed. The transitions into the states with $J_{z}= \pm 2$ are totally forbidden. The set of the states $\left\{\varphi_{i}\right\}$ specifies a basis of matrix representation of the $\mathrm{HH}$-exciton spin Hamiltonian. In the absence of the magnetic field, the matrix of the HH-exciton spin Hamiltonian is determined only by the exchange interaction and can be represented in the form

$$
H_{e-h}=\frac{1}{2}\left(\begin{array}{cccc}
\delta_{0} & \delta_{1} & 0 & 0 \\
\delta_{1} & \delta_{0} & 0 & 0 \\
0 & 0 & -\delta_{0} & \delta_{2} \\
0 & 0 & \delta_{2} & -\delta_{0}
\end{array}\right) \text {, }
$$

where $\quad \delta_{0}=-c_{z} / 4, \quad \delta_{1}=-\left(c_{x}+c_{y}\right) / 4, \quad$ and $\quad \delta_{2}=-\left(c_{x}\right.$ $\left.-c_{y}\right) / 4$.

The matrix of the Zeeman part of the Hamiltonian can be simplified by assuming axial symmetry of the $g$ factor. Note that, for the shape of the QD's under study, the value of the $g$ factor is controlled mainly by confinement along the growth axis. For this reason, asymmetry of the confinement in the plane of the QD's should not noticeably affect the Zeeman splitting. For axial symmetry, the Zeeman part of the Hamiltonian has the form

$$
H_{e}+H_{h}=H_{\text {Zeeman }}=\frac{\mu_{B} B}{2}\left(\begin{array}{cccc}
-\left(g_{e, z}+g_{h, z}\right) \cos \theta & 0 & g_{e, x} \sin \theta & g_{h, x} \sin \theta \\
0 & \left(g_{e, z}+g_{h, z}\right) \cos \theta & g_{h, x} \sin \theta & g_{e, x} \sin \theta \\
g_{e, x} \sin \theta & g_{h, x} \sin \theta & \left(g_{e, z}-g_{h, z}\right) \cos \theta & 0 \\
g_{h, x} \sin \theta & g_{e, x} \sin \theta & 0 & -\left(g_{e, z}-g_{h, z}\right) \cos \theta
\end{array}\right),
$$

where $\theta$ is the angle between the $z$ axis and direction of the magnetic field B. In Eq. (9), we used the coordinate system in which $B_{x}=B \sin \theta, B_{y}=0, B_{z}=B \cos \theta$.

The Hamiltonian matrices (8) and (9) contain a large number of uncertain parameters: three components of the exchange splitting, $\delta_{0,1,2}$, two components of the electron $g$ factor, $g_{e, z}$ and $g_{e, x}$, and two components of the hole $g$ factor, $g_{h, z}$ and $g_{h, x}$. To reduce the number of independent parameters, we use several approximations based on our experimental data.

It was noted above (see Fig. 3 and comments therein) that the beat frequency is proportional to the magnetic-field strength. This may occur only when the Zeeman splitting, in the range of the magnetic field employed, substantially exceeds the exchange one. Therefore we can neglect the exchange splitting assuming that $\delta_{0,1,2}=0$.

We can additionally simplify the spin Hamiltonian by assuming a strong anisotropy of the hole $g$ factor in the system under study, $g_{h, z} \gg g_{h, x}$. This approximation is used by most authors who analyze Zeeman splitting of exciton spins in semiconductor heterostructures. ${ }^{29,28}$ Thus, in the analysis of the beat frequency, we assume that $g_{h, x}=0$.

In the framework of the above approximations, the Hamiltonian matrices (8) and (9) contain only three free parameters $g_{e, z}, g_{e, x}$, and $g_{h, z}$. The energies of the Zeeman components can now be calculated analytically:

$$
E_{1,2,3,4}= \pm \frac{\mu_{B} B}{2}\left[g_{h, z} \cos \theta \pm\left(g_{e, z}^{2} \cos ^{2} \theta+g_{e, x}^{2} \sin ^{2} \theta\right)^{1 / 2}\right]
$$

The sign "- " inside the brackets corresponds to the states with energies $E_{1}$ and $E_{2}$ and the sign "+" to the states with energies $E_{3}$ and $E_{4}$.

The expressions for the wave functions obtained in an explicit analytical form are too cumbersome to be presented here.

\section{B. Quantum beats and fine structure}

Before using the expressions obtained in the previous subsection for theoretical analysis of the experimental results, we have to determine what particular states of the excitonic fine structure are responsible for the observed beats. There are two types of beats that can be observed in the states of the $\mathrm{HH}$ exciton split by the magnetic field: ${ }^{13}$

(i) The beats between sublevels of the optically active doublet, which are revealed as antiphase oscillations of the PL intensity in the linear co- and cross polarizations;

(ii) The beats between the bright and dark sublevels mixed by transverse component of the magnetic field.

We start the discussion with the beats of the second type because the beats in our experiments were observed only in 
tilted magnetic fields, i.e., in the presence of the transverse component of the field (see Sec. III).

\section{Beats between the bright and dark sublevels}

The transverse component of the magnetic field mixes states with different projections of the angular momentum, which makes transitions into optically inactive (dark) states partially allowed. In QD's, this effect was observed in Ref. 28. As follows from the Zeeman Hamiltonian matrix (9), the state $\varphi_{1}$ is mixed with the state $\varphi_{3}$, and the state $\varphi_{2}$ with $\varphi_{4}$. As a result, the circularly polarized light will simultaneously excite two components of the fine structure. In the absence of the spin relaxation, polarization of the PL from these components will reproduce polarization of excitation, and the PL intensity will oscillate with the frequency determined by the energy difference between these components. Thus under the $\sigma^{+}$polarized excitation the beats between the states $\varphi_{1}$ and $\varphi_{3}$ will show up, while under the $\sigma^{-}$polarized excitations, the beats between the states $\varphi_{2}$ and $\varphi_{4}$. In both cases, the beats should appear in the co-polarized PL.

The circular cross-polarized PL may appear only after the excitonic spin-flip process occurs. ${ }^{8}$ Since the spin flip completely destroys the spin coherence, the beats of the above type cannot be observed in the cross-polarized PL.

The whole set of the features mentioned above (the presence of the beats in the circular co-polarized PL, their absence in the cross-polarized PL, and the necessity of the transverse component of the magnetic field) is present in the beats we observed (see Sec. III). Thus we can conclude that these beats are really related to the interference between the bright and dark states. The frequency of the beats is determined, in this case, by the energy difference between the relevant Zeeman components, i.e., by the quantity

$$
\hbar \omega=E_{1}-E_{3}=\mu_{B} B\left(g_{e, z}^{2} \cos ^{2} \theta+g_{e, x}^{2} \sin ^{2} \theta\right)^{1 / 2} .
$$

Equation (11) contains only longitudinal $g_{e, z}$ and transverse $g_{e, x}$ components of the electron $g$ factor. Partial contributions of the components depend on the tilt angle $\theta$. This makes it possible to unambiguously determine these components from the experimental data. For comparison with the experiment, we calculated dependences of the QB's frequencies, observed in the $\sigma^{+}$polarization, on the angle $\theta$, and magneticfield strength. The calculations were made using expression (11), with the quantities $g_{e, z}$ and $g_{e, x}$ regarded as fitting parameters. The results of the comparison of the theory and experiment are shown in Figs. 3(b) and 4(b). As is seen, one set of the parameters $\left(g_{e, z}=0.53\right.$ and $\left.g_{e, x}=1.43\right)$ allows us to describe well all the experimental dependences.

An attempt to refine the model with allowance for the isotropic component of the exchange splitting $\delta_{0}$ has not been successful. It was found that introducing the exchange coupling $\delta_{0} \leqslant 30 \mu \mathrm{eV}$ practically did not affect the results of the calculations. At greater $\delta_{0}$, the agreement between the calculated and experimental dependences was rapidly worsening. Based on these facts, we concluded that the exchange coupling energy in the structure under study does not exceed $30 \mu \mathrm{eV}$.

\section{Splitting of the bright doublet}

The experimental fact of absence of the beats between the bright doublet components in a longitudinal magnetic field (see Sec. III) needs to be specially discussed. It immediately follows from the spin Hamiltonian (8) that the longitudinal magnetic field splits the optically active doublet $\left(J_{z}= \pm 1\right)$ into two components $E_{1}$ and $E_{2}$, with the splitting determined by the longitudinal component of the exciton $g$ factor, $g_{z}=g_{e, z}+g_{h, z}: \Delta E=\mu_{B} B g_{z}$. The coherent excitation of the split states with the linearly polarized light should give rise to quantum beats in the polarized PL at a frequency of $\omega$ $=\delta E / \hbar$. Such beats are easily observed in the experiments with the GaAs quantum wells. ${ }^{27,8}$

The main reason for the absence of the beats in our case is, most likely, a large spread of the splittings of the bright doublet in the QD's ensemble. The spread may be predominantly caused by the spread of the longitudinal components of the excitonic $g$ factor. Indeed, according to the experimental data for single InP QD's obtained in Ref. 7, the spread of the longitudinal $g$ factor is very large and makes up $\Delta g_{z}$ $= \pm 0.5$.

A result of the spread of the splittings should be destructive interference between the beat signals from different QD's. At small spread, such an interference leads to acceleration of decay of the beat signal (reversible dephasing).$^{32}$ If the spread substantially exceeds the mean value of the splitting, the beats cannot be observed and the degree of polarization of the PL decays smoothly in time. For the Gaussian spread of the splittings, the spread of the beat frequencies $\delta \omega$ and the decay time $\tau$ are connected by a simple relationship: $\delta \omega=2 / \tau$. In our experiments, we indeed observed the smooth decay of the degree of linear polarization in the longitudinal magnetic field. The decay time in the field $B$ $=1.5 \mathrm{~T}$ makes up approximately $20 \mathrm{ps}$, which corresponds to the spread $\Delta g_{z} \approx 0.7$. This value is comparable with the one given in Ref. 7. The absence of the beats means that the mean value of $g_{z}$, in our sample, is significantly smaller than the spread, i.e., $\left\langle g_{z}\right\rangle<0.7$.

Thus, due to the large spread of the excitonic $g$ factor, the beats between the Zeeman components of the bright doublet are not observed. At the same time, this spread does not prevent from observing the beats between the dark and bright states, described in the previous section. This apparent paradox can be easily explained with allowance for the fact that the splitting between the dark and bright components is controlled by the electron $g$ factor only [see Eq. (11)], whereas the splitting of the bright doublet is determined by the sum of the electron and hole $g$ factors. Hence it follows that the spread of the electron $g$ factor in the QD's under study is rather small and the main contribution to the spread of the excitonic $g$ factor is made by the hole $g$ factor. The reason for such a large spread of the hole $g$ factors calls for further investigation.

\section{Oscillation amplitude}

The calculations fulfilled in the framework of the spinHamiltonian model has allowed us to determine not only the frequencies but also the whole shape of oscillations of the 
polarized PL in the magnetic field. To describe the shape of the oscillations, we calculated the time-dependent matrix elements of optical transitions from the relevant states of the fine structure.

The eigenfunctions of the spin-Hamiltonian in a magnetic field of arbitrary orientation are linear combinations of the basis functions $\left\{\varphi_{i}\right\}=\{|+1\rangle,|-1\rangle,|+2\rangle$, and $|-2\rangle\}$ :

$$
\psi_{i}=\sum_{j=1}^{4} a_{i j} \varphi_{j} .
$$

The expansion coefficients $a_{i j}$ can be found by solving the stationary Schrödinger equation with Hamiltonian (8) and (9). The coherent pulsed excitation creates, at the initial moment, a linear superposition of the states $\psi_{i}$, whose subsequent evolution is described by the equation

$$
\Psi(t)=\sum_{i=1}^{4} C_{0 i} \exp \left(-i E_{i} t / \hbar\right) \psi_{i} .
$$

Here, $C_{0, i}$ are the time-independent coefficients whose values are determined by the initial conditions of excitation.

In accordance with the selection rules for optical transitions, the $\sigma^{+}$polarized light excites each of the eigenstates $\psi_{i}$ in proportion with the admixture of the basis function $|+1\rangle$. The mixing is determined by the coefficients $a_{i j}$ with $j=1$ in Eq. (12), so that the relationship $C_{0 i}=a_{i 1}^{*}$ is fulfilled.

The PL intensity in the $\sigma^{+}$polarization $I_{+}$is proportional to the matrix element of the optical transition squared:

$$
I_{+} \propto|\langle\Psi(t)|\hat{d}| 0\rangle|^{2},
$$

where $\hat{d}$ is the dipole moment operator and $|0\rangle$ is the ground state of the system. From Eqs. (13) and (14) and the expression for the coefficients $C_{0 i}$ given above, we obtain the following expression for the PL signal (to within a constant factor):

$$
I_{+}=\sum_{i}^{4}\left|a_{i 1}\right|^{4}+2 \sum_{i<k}^{4}\left|a_{i 1}\right|^{2}\left|a_{k 1}\right|^{2} \cos \left(\frac{E_{i}-E_{k}}{\hbar} t\right) .
$$

The first sum in Eq. (15) describes the smooth component of the signal $I_{s}$. It does not contain any time dependence since the model we use does not take into account relaxation processes. The oscillating part of the signal is described by the second sum in Eq. (15). In the framework of the above approximations, the only nonzero coefficients in this term, for the $\sigma^{+}$polarized excitation, are $a_{1,1}$ and $a_{3,1}$.

Thus the expression for the beats intensity $I_{\text {beats }}$ (see definition in Sec. III) can be reduced to the form

$$
I_{\text {beats }}=\frac{2 R \sum_{i<k}^{4}\left|a_{i 1}\right|^{2}\left|a_{k 1}\right|^{2} \cos \left(\frac{E_{i}-E_{k}}{\hbar} t\right)}{\sum_{i}^{4}\left|a_{i 1}\right|^{4}} \exp (-t / \tau) .
$$

The amplitude factor $R$ is introduced to take into account the loss of coherence in the process of relaxation of the photo-

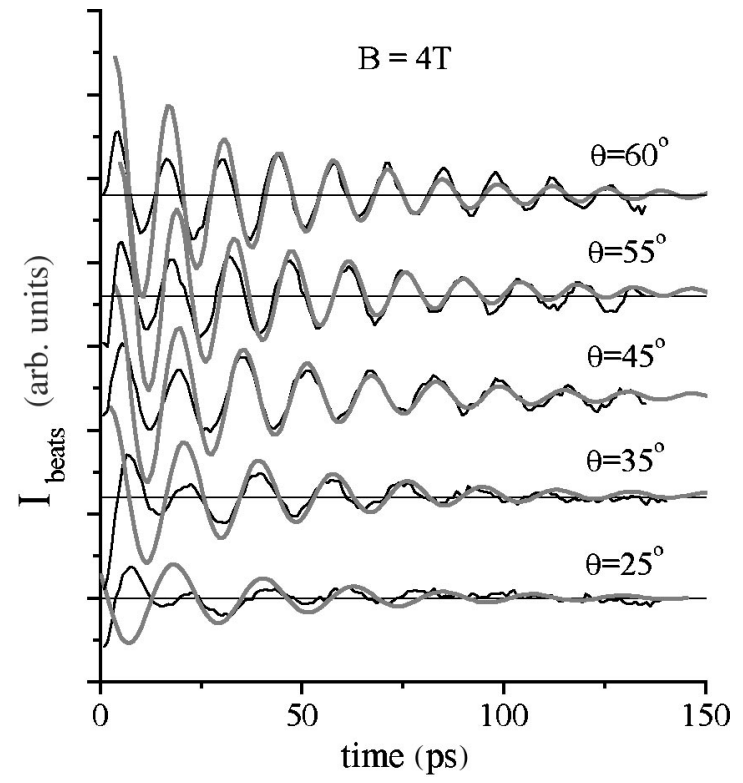

FIG. 5. Quantum beats in the InP QD's in a magnetic field of $B=4 \mathrm{~T}$. The noisy curves are the experiment and the thick gray lines are the calculations.

generated electron-hole pairs to the radiative energy level. ${ }^{33}$ In addition, Eq. (16) contains a phenomenological exponential factor that takes into account the decay of the beats. Equation (16) allows us to determine the Zeeman splittings $E_{i}-E_{k}$ and decay constant $\tau$ from the experimental data.

Analyzing our experimental data, we have found that the beat decay rate $1 / \tau$ depends on orientation of the magnetic field. An increase in the angle $\theta$, i.e., a growth of the transverse component $B_{x}$, is accompanied by an increase in the beat decay rate. This effect is most likely to be related to the presence of a small transverse component $g_{h, x}$ of the hole $g$ factor. As follows from the spin Hamiltonian (9), the transverse component of the magnetic field, at a nonzero $g_{h, x}$, admixes each bright state to the both dark states, rather than only to one of them. As a result, the beats arise at several frequencies. The interference of these beats is revealed as a decay of the main harmonic. Since the value of the admixture is proportional to the square of the transverse component of the magnetic field, this effect should be observed only at large angles $\theta$.

Figure 5 shows an example of comparison of the experimental data with the results of calculations for the angles $\theta$ $\leqslant 60^{\circ}$ and magnetic field $B=4 \mathrm{~T}$. The calculations were made using formula (16) with allowance for an additional mixing of the states due to nonzero $g_{h, x}$. The value $g_{h, x}$ was used in the calculations as one more fitting parameter. It has been found that the best agreement with the experiment is achieved at $g_{h, x}=0.1$. It is important that all the experimental data obtained in the whole range of the magnetic-field strengths and for the angles $\theta \leqslant 60^{\circ}$ can be well fitted using one set of the parameters: $g_{e, z}=0.53, g_{e, x}=1.43, R=0.8$, and $\tau=40 \mathrm{ps}$. It should be emphasized that the estimated value of the $x$ component of the hole $g$ factor $g_{h, x}$ is small with respect to the electron $g$ factor. Therefore this component virtually does not affect the beat frequency. 
A good agreement it is worth noting between the calculations and experimental data in spite of a limited number of parameters. A certain discrepancy between the calculations and experiments is observed only at the initial moment $(<10 \mathrm{ps})$. This is probably related to the error of modeling of the smooth background upon extracting the beats from the total signal. ${ }^{34}$

It is surprising that the value $R$ is rather large. It means that about $80 \%$ of the coherence produced by the excitation is conserved after relaxation of the electron-hole pair to the radiative energy level. In this respect, the situation in the structure with quantum dots under study essentially differs from that in quantum wells, where, according to Ref. 13, the energy relaxation by more than $20 \mathrm{meV}$ is accompanied by complete loss of the hole spin orientation. In our case, it is probably highly important that the relaxation occurs with emission of an LO phonon, and such a process is very fast. The relaxation with emission of acoustic phonons conserves a much smaller fraction of the coherence. ${ }^{26}$

The above set of the parameters makes it possible to adequately describe the shape of the oscillating signal at the tilt angles up to $\theta \leqslant 60^{\circ}$. At larger angles, the oscillation amplitude sharply decreases (see Sec. III) and, to obtain agreement with the experiment, one has to significantly change the amplitude factor $R$. The reason for this effect invites further studies.

The proposed theory, as a whole, describes adequately the behavior of the beat amplitudes and frequencies at different values and orientations of the magnetic field. At the same time, a few questions remain open, calling for further research. First of all, the great difference between components of the electron $g$ factor, $\left(g_{e, x}-g_{e, z}\right) \approx 0.9$, is fairly unusual. This value in quasi-two-dimensional heterostructures lies, as a rule, in the range of $0.1-0.2 \cdot{ }^{35,36}$ On the other hand, the value $g_{e, x}$ we obtained practically coincides with that of the electronic $g$ factor in the bulk InP. ${ }^{37}$ Therefore we have to admit strong suppression of the longitudinal component of the electron $g$ factor in the InP QD's.

One more curious fact is that the exchange splitting in the structure under study is rather small, $\delta_{0}<30 \mu \mathrm{eV}$. Our estimate of $\delta_{0}$ strongly differs from the value $\delta_{0} \approx 100 \mu \mathrm{eV}$ found experimentally for the negatively charged exciton, trion, at the same QD's. ${ }^{17}$ Such a strong difference between the exchange coupling in trion and in neutral exciton calls for special theoretical analysis.

\section{CONCLUSIONS}

In this study, we observed quantum beats between Zeeman components of the fine structure of electron-hole pairs in the InP quantum dots. This proved to be possible after removal of excess charges upon application of negative electric bias to the top surface of the sample. It was found that the beats show specific polarization characteristics and depend in a nontrivial way on the magnetic-field orientation. Analysis of the experimental data within the framework of the spin-Hamiltonian model has allowed us to consistently explain the observed phenomena and to quantitatively describe the shape of the quantum beats signal. As a result, we have determined the values of the transverse and longitudinal components of the electron $g$ factor $\left(g_{e, z}=0.53\right.$ and $g_{e, x}$ $=1.43)$ and estimated the exchange coupling $\left(\delta_{0}\right.$ $<30 \mu \mathrm{eV}$ ). Based on the analysis of the experimental data, we came to the conclusion about a considerable spread of the hole $g$ factor $\left(\Delta g_{h} \approx 0.7\right)$ and about nonzero value of the transverse component $\left(g_{h, x} \approx 0.1\right)$ of the hole $g$ factor.

\section{ACKNOWLEDGMENTS}

The authors wish to thank Professor V. Zapasskii, Dr. G. Kozlov, and Dr. K. Kavokin for a fruitful discussion.
*Electronic address: irina.yugova@ pobox.spbu.ru

†Permanent address: Vavilov State Optical Institute, St.-Petersburg, 190034, Russia.

${ }^{\ddagger}$ Permanent address: Institute of Physics, St.-Petersburg State University, St.-Petersburg, 198504, Russia.

${ }^{\S}$ Permanent address: Institute of Physics, St.-Petersburg State University, St.-Petersburg, 198904, Russia.

IPresent address: Applied Optoelectronics Incorporation, Sugar Land, TX 77478.

**Permanent address: Faculty of Science, Osaka City University, Osaka 558-8585, Japan.

${ }^{\dagger}$ Permanent address: Opto-Electronic Research Laboratory, NEC Corporation, Tsukuba, 305-8501, Japan.

\$Permanent address: Institute of Physics, University of Tsukuba, Tsukuba 305-8571, Japan.

${ }^{1}$ D.D. Awschalom and J.M. Kikkawa, Phys. Today 52(6), 33 (1999).

${ }^{2}$ E. Pazy, I. D’Amico, P. Zanardi, and F. Rossi, Phys. Rev. B 64, 195320 (2001).

${ }^{3}$ D. Loss and D.P. DiVincenzo, Phys. Rev. A 57, 120 (1998).

${ }^{4}$ M. Paillard, X. Marie, P. Renucci, T. Amand, A. Jbeli, and J.M. Gerard, Phys. Rev. Lett. 86, 1634 (2001).
${ }^{5}$ M. Baier, F. Findeis, A. Zrenner, M. Bichler, and G. Abstreiter, Phys. Rev. B 64, 195326 (2001).

${ }^{6}$ M. Bayer, A. Kuther, A. Forchel, A. Gorbunov, V.B. Timofeev, F. Schäfer, J.P. Reithmaier, T.L. Reinecke, and S.N. Walck, Phys. Rev. Lett. 82, 1748 (1999).

${ }^{7}$ M. Sugisaki, H.W. Ren, K. Nishi, S. Sugou, T. Okuno, and Y. Masumoto, Physica B 256-258, 169 (1998).

${ }^{8}$ I.Ya. Gerlovin, Yu.K. Dolgikh, S.A. Eliseev, V.V. Ovsyankin, Yu.P. Efimov, V.V. Petrov, I.V. Ignatiev, I.E. Kozin, and Y. Masumoto, Phys. Rev. B 65, 035317 (2002).

${ }^{9}$ S. Bar-Ad and I. Bar-Joseph, Phys. Rev. Lett. 66, 2491 (1991).

${ }^{10}$ A.P. Heberle, W.W. Ruhle, and K. Ploog, Phys. Rev. Lett. 72, 3887 (1994).

${ }^{11}$ R.E. Worsley, N.J. Traynor, T. Grevatt, and R.T. Harley, Phys. Rev. Lett. 76, 3224 (1996).

${ }^{12}$ L. Sham, Science 277, 1258 (1997).

${ }^{13}$ T. Amand, X. Marie, P. Le Jeune, M. Brousseau, D. Robart, J. Barrau, and R. Planel, Phys. Rev. Lett. 78, 1355 (1997).

${ }^{14}$ J.A. Gupta, D.D. Awschalom, X. Peng, and A.P. Alivisatos, Phys. Rev. B 59, R10 421 (1999).

${ }^{15}$ V.K. Kalevich, M.N. Tkachuk, P. Le Jeune, X. Marie, and T. Amand, Fiz. Tverd. Tela 41, 871 (1999) [Phys. Solid State 41, 789 (1999)]. 
${ }^{16}$ T. Flissikowski, A. Hundt, M. Lowisch, M. Rabe, and F. Henneberger, Phys. Rev. Lett. 86, 3172 (2001).

${ }^{17}$ I.E. Kozin, V.G. Davydov, I.V. Ignatiev, A.V. Kavokin, K.V. Kavokin, G. Malpuech, H.W. Ren, M. Sugisaki, S. Sugou, and Y. Masumoto, Phys. Rev. B 65, 241312(R) (2002).

${ }^{18}$ R.J. Warburton, C.S. Durr, K. Karrai, J.P. Kotthaus, G. MedeirosRibeiro, and P.M. Petroff, Phys. Rev. Lett. 79, 5282 (1997).

${ }^{19}$ A. Hartmann, Y. Ducommun, E. Kapon, U. Hohenester, and E. Molinari, Phys. Rev. Lett. 84, 5648 (2000).

${ }^{20}$ Dan Hessman, Jonas Persson, Mats-Erik Pistol, Craig Pryor, and Lars Samuelson, Phys. Rev. B 64, 233308 (2001).

${ }^{21}$ H.-W. Ren, M. Sugisaki, S. Sugou, K. Nishi, A. Gomyo, and Y. Masumoto, Jpn. J. Appl. Phys., Part 1 38, 2438 (1999).

${ }^{22}$ Y. Masumoto, V. Davydov, I. Ignatiev, H.-W. Ren, and S. Sugou, Jpn. J. Appl. Phys., Part 1 38, 563 (1999).

${ }^{23}$ I.V. Ignatiev, I.E. Kozin, V.G. Davydov, S.V. Nair, J.-S. Lee, H.-W. Ren, S. Sugou, and Y. Masumoto, Phys. Rev. B 63, 075316 (2001).

${ }^{24}$ Y. Masumoto, I.V. Ignatiev, I.E. Kozin, V.G. Davydov, S.V. Nair, H.-W. Ren, J.-S. Lee, and S. Sugou, Jpn. J. Appl. Phys., Part 1 40, 1947 (2001).

${ }^{25}$ In Ref. 17 it has been shown that at a voltage of $U=-0.2 \mathrm{~V}$ each quantum dot contains, on average, one resident electron, the electron distribution being described by a Gaussian with a half width at half maximum of $\Delta U=0.22 \mathrm{~V}$. With increasing negative voltage $(U<-0.2 \mathrm{~V})$, the number of neutral quantum dots increases, while with increasing positive voltage ( $U$ $>-0.2 \mathrm{~V})$, the number of multiply charged dots increases. Thus we can easily obtain the expression for the dependence of the number of uncharged quantum dots on the voltage, used to fit the data in Fig. 1(d).

${ }^{26}$ I.A. Yugova, V.G. Davydov, I.Ya. Gerlovin, I.V. Ignatiev, I.E. Kozin, M. Sugisaki, and Y. Masumoto, Phys. Status Solidi A
190, 547 (2002).

${ }^{27}$ R.E. Worsley, N.J. Traynor, T. Grevatt, and R.T. Harley, Phys. Rev. Lett. 76, 3224 (1996).

${ }^{28}$ M. Bayer, O. Stern, A. Kuther, and A. Forchel, Phys. Rev. B 61, 7273 (2000).

${ }^{29}$ H.W. van Kesteren, E.C. Cosman, W.A.J.A. van der Poel, and C.T. Foxon, Phys. Rev. B 41, 5283 (1990).

${ }^{30}$ E.L. Ivchenko and G.E. Pikus, Superlattices and Other Heterostructures Symmetry and Optical Phenomena, Springer Series in Solid-State Sciences Vol. 110 (Springer, Berlin, 1997).

${ }^{31}$ E.L. Ivchenko and A.A. Kiselev, Fiz. Tekh. Poluprovodn. 26, 1471 (1992) [Sov. Phys. Semicond. 26, 827 (1992)].

${ }^{32}$ H. Nickolaus, H.-J. Wunsche, and F. Henneberger, Phys. Rev. Lett. 81, 2586 (1998).

${ }^{33} \mathrm{~A}$ possible reason for the decrease in the beats signal can also be incomplete orientation of spins of the electron-hole pair because of mixing of excited states of the light and heavy holes. As a result, e.g., under the $\sigma^{+}$-polarized excitation, the electrons, both with the spin $s=+1 / 2$ and with the spin $s=-1 / 2$, can be generated. A certain role can be also played by ellipticity of the exciting light arising upon oblique incidence of the beam upon the sample.

${ }^{34}$ The processes of formation of the secondary emission at the initial stages are rather complicated [see, i.e., W. Langbein, K. Leosson, J.R. Jensen, J.M. Hvam, and R. Zimmermann, Phys. Rev. B 61, R10 555 (2000)] and may also result in behavior of the beats other than that described by Eq. (1).

${ }^{35}$ E.L. Ivchenko, Pure Appl. Chem. 67, 463 (1995)

${ }^{36}$ A. Malinowski and R.T. Harley, Phys. Rev. B 62, 2051 (2000).

${ }^{37}$ Semiconductors, Intrinsic Properties of Group V Elements and III-V, II-VI, and I-VI Compounds, edited by K.-H. Hellwege and O. Madelung, Landolt-Börnstein, New Series, Group III, Vol. 22, Pt. a (Springer-Verlag, Berlin, 1987). 